\title{
CoreSlicer: a web toolkit for analytic morphomics
}

Louis Mullie ${ }^{1,2}$ and Jonathan Afilalo $0^{1,2,3^{*}}$

\begin{abstract}
Background: Analytic morphomics, or more simply, "morphomics," refers to the measurement of specific biomarkers of body composition from medical imaging, most commonly computed tomography (CT) images. An emerging body of literature supports the use of morphomic markers measured on single-slice CT images for risk prediction in a range of clinical populations. However, uptake by healthcare providers been limited due to the lack of clinician-friendly software to facilitate measurements. The objectives of this study were to describe the interface and functionality of CoreSlicer- a free and open-source web-based interface aiming to facilitate measurement of analytic morphomics by clinicians - and to validate muscle and fat measurements performed in Coreslicer against reference software.

Results: Measurements of muscle and fat obtained in CoreSlicer show high agreement with established reference software. CoreSlicer features a full set of DICOM viewing tools and extensible plugin interface to facilitate rapid prototyping and validation of new morphomic markers by researchers. We present published studies illustrating the use of CoreSlicer by clinicians with no prior knowledge of medical image segmentation techniques and no formal training in radiology, where CoreSlicer was successfully used to predict operative risk in three distinct populations of cardiovascular patients.
\end{abstract}

Conclusions: CoreSlicer enables extraction of morphomic markers from CT images by non-technically skilled clinicians. Measurements were reproducible and accurate in relation to reference software.

Keywords: Analytic morphomics, Morphometric analysis, Body composition analysis, Planimetric measurements, Medical image segmentation, Computed tomography, Obesity, Sarcopenia

\section{Background}

Syndromes characterized by pathological alterations of body composition, such as sarcopenia, cachexia and obesity, are increasingly prevalent and portend an increased risk of adverse health outcomes. Accordingly, there is a growing interest, both at the clinical and academic levels, in using body composition analysis to identify vulnerable patients who would benefit from targeted evaluation and treatment [1]. Analytic morphomics, or more simply, "morphomics," refers to the measurement of specific biomarkers of body composition from medical imaging, most commonly computed tomography (CT) images [2-4]. An emerging body of literature

\footnotetext{
* Correspondence: jonathan.afilalo@mcgill.ca

${ }^{1}$ Department of Medicine, McGill University, 3755 Cote Ste Catherine Rd, E-222, Montreal, QC H3T 1E2, Canada

${ }^{2}$ Division of Cardiology, McGill University, Montreal, QC, Canada

Full list of author information is available at the end of the article
}

supports the use of morphomic markers measured on single-slice CT images for risk prediction in a range of clinical populations (Table 1). The analytic morphomics group at the University of Michigan has had a pioneering influence in the field and has undertaken a major standardization effort with the publication of reference values derived from a large population of patients [5]. Despite promising results, adoption by healthcare providers has been limited due to the lack of clinicianfriendly software to facilitate measurements.

Figure 1 displays a typical workflow for determination of morphomic markers; which begins by opening a study in DICOM format, selecting a validated reference anatomical level on a reconstructed view (e.g. the level of the 4th lumbar vertebra on a sagittal view), visualizing the corresponding axial image, and finally performing measurements using a combination of automatic and manual segmentation tools. Markers that can be

(c) The Author(s). 2019 Open Access This article is distributed under the terms of the Creative Commons Attribution 4.0 International License (http://creativecommons.org/licenses/by/4.0/), which permits unrestricted use, distribution, and 
Table 1 Selected single-slice morphomic markers derived from muscle and adipose tissue area measurements on thoracic and abdominal CT scans

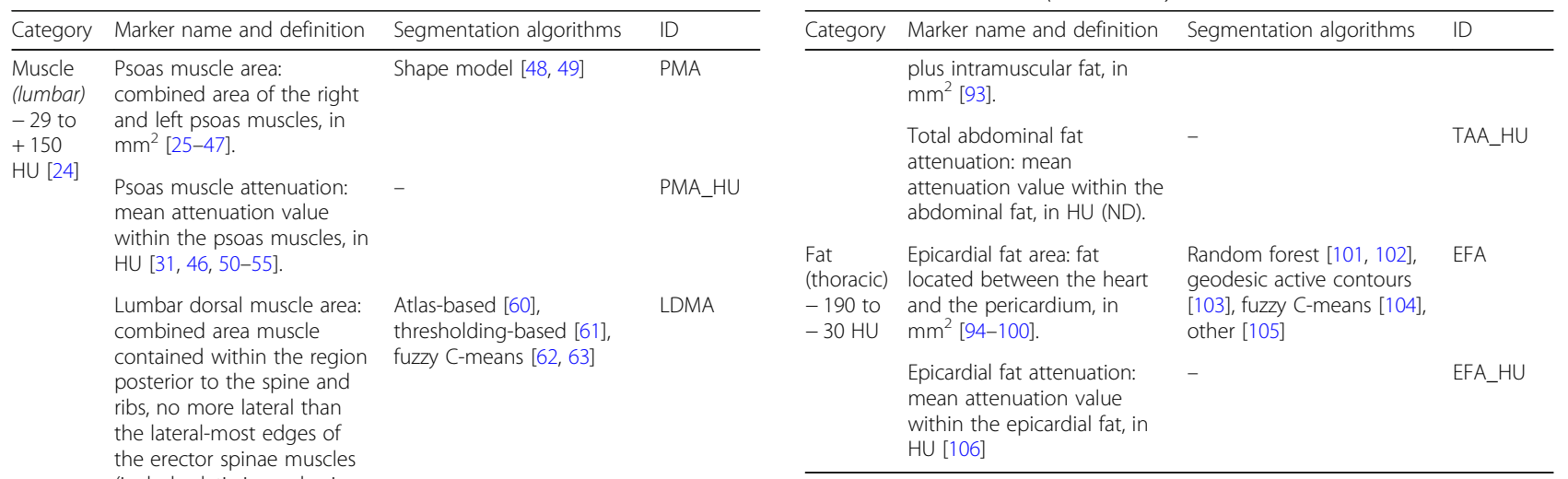

Table 1 Selected single-slice morphomic markers derived from muscle and adipose tissue area measurements on thoracic and abdominal CT scans (Continued)

Total thoracic muscle attenuation: mean

attenuation value within the lumbar muscles, in HU (ND).

Fat Visceral fat area: total area of (lumbar) intraperitoneal fat, in $\mathrm{mm}^{2}$

-190 to $\quad[31,54,55,75-79]$.

$-30 \mathrm{HU}$

FEM-based $[67,68]$

TLMA

TLMA_HU

FEM-based [74]

Fuzzy C-means [80], fuzzy affinity [81], thresholding [82], separation mask [83] polar projection ([84], edge linking [85], other [86]

Visceral fat attenuation: mean attenuation value within the visceral fat, in $\mathrm{HU}$ [51, 53, 87-93].

Subcutaneous fat area: total area of fat tissue between the skin and abdominal/ back wall, in $\mathrm{mm}^{2}[31,51$, 54, 55].

Subcutaneous fat attenuation: mean attenuation value within the subcutaneous fat, in $\mathrm{HU}$ [53, 87-91].

Total abdominal fat area: combined area of visceral and subcutaneous fat tissue,

extracted using this workflow include tissue areas (muscle, fat, solid organs, bone), as well as attenuation values in Hounsfield units (HU), which provide an index of tissue composition or "quality." Key advantages over other methods of body composition assessment include the wide availability of existing CT datasets, the ability to perform measurements retrospectively, and the ability to accurately assess tissue quantity, quality and distribution with high reproducibility and minimal assumptions.

Table 1 displays selected examples of morphomic markers of muscle and adipose tissue derived from single-slice measurements on abdominal or thoracic CT scans, with accompanying references to clinical validation studies. The wide range of applications illustrates the versatility of the proposed workflow. Automated segmentation algorithms are referenced when available.

Given the increasing availability of clinically indicated CT imaging studies [6] and the mounting recognition of morphomics as a prognostically relevant method of body composition assessment, it is highly desirable to facilitate measurements for researchers and clinicians. However, developing software for the morphomics community poses unique development challenges because the field exists at the confluence of multiple scientific disciplines, including medical image analysis (MIA), body composition research, clinical medicine, and epidemiology. We have identified four key challenges and corresponding design requirements for software to bring morphomics "to the bedside" and to a larger community of researchers (Table 2). In this section, we review existing MIA software in light of these design constraints and identify pertinent limitations of established approaches.

A first key challenge is to bridge the gap in technical knowledge between body composition researchers, who develop and validate morphomic markers, and healthcare providers who can utilize these markers to enhance their evaluation and treatment of patients. The ability quickly review and edit results is crucial in the clinical 


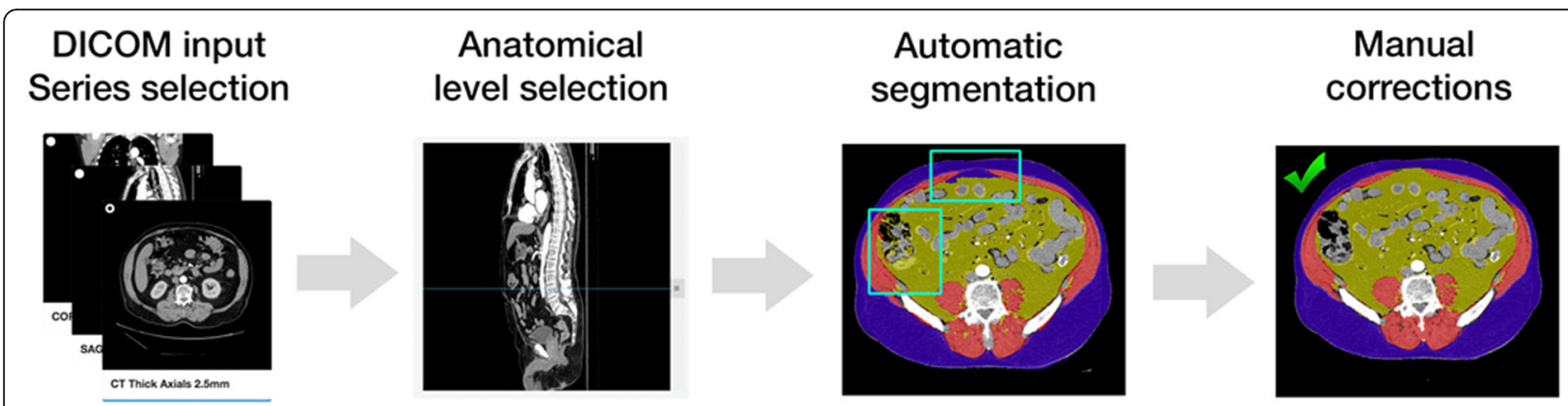

Fig. 1 Typical workflow for measurement of analytic morphomics

setting, where expert validation of computer-generated data is considered standard of care. Thus, for morphomics data to translate into improved decision-making, a streamlined, "clinician-friendly" interface for reviewing results is essential.

Current MIA software packages have adopted the "toolbox" approach of user interface organization. This approach is flexible, yet results in a high level of interface complexity due to the multiplicity of controls and menus. For example, the main user control interface includes 27 visible clickable control elements in Nora [7], 28 in Image [8], and 32 in slice-O-Matic [9]. By contrast, the "wizard" approach is an organizational pattern that presents essential controls in step-by-step fashion, which facilitates the execution of unfamiliar tasks. The wizard or "workflow-oriented" approach is most useful for "a non-expert user [who] needs to perform an infrequent complex task consisting of several subtasks, where decisions need to be made in each subtask." [10] While the workflow-oriented pattern may be a valuable approach for clinician-facing MIA software, only one of the major packages evaluated has adopted this approach as a primary user interface organization pattern, and this software did not support workflows for measurement of analytic morphomics [11].

A second key challenge is cross-platform compatibility, which is crucial to address owing to the heterogeneity in computing environments deployed by teams of researchers and clinicians working across different institutions. Of the major currently existing tools, Slice-O-matic, Materialize [12] and Segment [13] only offer Windows support, while Osirix only supports Mac environments [14]. 3DSlicer [15], ImageJ [15] and ITKSnap [16] are notable free options with good cross-platform compatibility. Although extremely powerful, their interfaces have not been adapted to facilitate measurements by non-clinicians with little technical knowledge in image analysis.

Most current MIA software packages (e.g. ImageJ, Segment, Osirix, 3DSlicer) are distributed via desktop apps that are installed on end-user machines, and these require continuous updating to ensure forward-compatibility. Yet, clinicians may be performing measurements on work machines where MIA software has not been installed, and, in many cases, institutional restrictions prevent end-users from installing such software in the clinical environment. Over the last decade, browser applications, or "web apps," have emerged as a more sustainable means of achieving robust cross-platform independence; these can be used instantly on any machine connected to the Internet, without prior installation. Nora is an example of an MIA software with an emphasis on brain imaging that is written entirely as a browser application [7]. Nora provides many advanced analysis features; limitations of this project are the lack of a plugin architecture, moderate to high interface complexity, and lack of a formal open-source development process.

A third challenge is to facilitate prototyping, validation and adoption of algorithms to segment scans and

Table 2 Design objectives for translational morphomics software

\begin{tabular}{ll}
\hline Design objective & Rationale \\
\hline Clinician-friendly, goal-directed interface & $\begin{array}{l}\text { Clinicians may not have the time and technical know-how required to use professional medical image } \\
\text { analysis software. }\end{array}$ \\
$\begin{array}{ll}\text { Cross-platform support, minimal or no } \\
\text { install required }\end{array}$ & $\begin{array}{l}\text { Researchers and clinicians collaborating on morphomics projects across institutions are likely to work in } \\
\text { different computer system environments. Clinicians may be performing measurements on work machines } \\
\text { where MIA software has not been installed. }\end{array}$ \\
$\begin{array}{ll}\text { Extensibility via cloud-enabled plugins } \\
\text { Free license and open source codebase plugin interface enables application of the software to a wider variety of use cases, and cloud } \\
\text { abilities facilitate the processing of large datasets. }\end{array}$ & $\begin{array}{l}\text { An open-source codebase and reuse-friendly license contributes to project sustainability by allowing } \\
\text { contributions from other researchers. }\end{array}$ \\
\hline
\end{tabular}


measure morphomic markers. Notably, at the time of writing, none of the segmentation algorithms presented in Table 1 had been released as a plugin compatible with freely available MIA software. While some MIA software have support for language-specific plugins (e.g. Java for ImageJ, Objective $\mathrm{C}$ for Osirix, Matlab for Segment, Python and $\mathrm{C}++$ for 3DSlicer), the lack of cross-talk between these languages hinders the development of a robust plugin ecosystem. A modern method of providing extensibility via "plugins" is the use of HTTP application programming interfaces (APIs), which enable the developer to write plugins in any language capable of running a simple web server. As an added benefit, computational resources in the cloud can be leveraged with the same interface and ease of use as local plugins. The neuroimaging community has already adopted cloud-based tools such as MRIcloud [17], CBRAIN [18] and VolBrain [19] to facilitate collaboration and sharing of computational resources across geographically dispersed research groups. Given the large-scale nature of the data sets used in morphomics, the community would benefit from a platform that enables rapid prototyping and deployment of morphomics algorithms in the cloud.

Finally, a free licensing model and an open-source development process was chosen as a design goal to facilitate greater adoption of the software amongst researchers, and to promote project sustainability by allowing developers to contribute their own updates and plugins. Given that the adoption of commercial MIA software imposes a financial limit on wider-scale collaboration, it is in the multidisciplinary spirit of the morphomics community to involve all stakeholders in building free, open-source alternatives that are equitably available to a global audience of clinicians irrespective of cost.

As summarized in Table 3, a review of existing MIA software identified that none satisfactorily addressed all of the key design constraints that we have proposed to guide development of translational morphomics software. While many are comprehensive and powerful tools, none of the currently available software tools provides a simple, clinician-friendly pipeline to facilitate morphomic analysis. To address this unmet need, we introduce the first web-based interface optimized for measurement of morphomics, called CoreSlicer, which is publicly available free of charge at https://www.coreslicer.com. Users can extend the software with their own plugins running on their own machine or on cloud services through HTTP endpoints. The source code to the interface is available on GitHub, and allows users to run the application on their local machines. In this paper, we discuss the structure and functionality of CoreSlicer, validate its results against reference software, and discuss published studies illustrating its relevance for clinicians and researchers.

Table 3 Selected major medical image analysis tools potentially suitable for morphomic analysis, features and limitations

\begin{tabular}{|c|c|c|c|c|c|c|c|}
\hline Project name and URL & $\begin{array}{l}\text { Workflow- } \\
\text { oriented }\end{array}$ & $\begin{array}{l}\text { Web } \\
\text { interface }\end{array}$ & $\begin{array}{l}\text { Platform } \\
\text { independent }\end{array}$ & $\begin{array}{l}\text { Plugin } \\
\text { interface }\end{array}$ & $\begin{array}{l}\text { Web } \\
\text { plugins }\end{array}$ & $\begin{array}{l}\text { Free } \\
\text { license }\end{array}$ & $\begin{array}{l}\text { Open } \\
\text { source }\end{array}$ \\
\hline $\begin{array}{l}\text { Slice-o-matic } \\
\text { http://www.tomovision.com/products/ } \\
\text { sliceomatic.html }\end{array}$ & $\mathrm{N}$ & $\mathrm{N}$ & N (Windows-only) & $N$ & $N$ & $N$ & $N$ \\
\hline $\begin{array}{l}\text { ImageJ } \\
\text { https://imagej.nih.gov/ij/ }\end{array}$ & $\mathrm{N}$ & N & Y (Java app) & Java only & $\mathrm{N}$ & Y & Y \\
\hline $\begin{array}{l}\text { Materialize } \\
\text { http://www.materialise.com/en/medical/ } \\
\text { software/mimics }\end{array}$ & N & N & N(Windows-only) & N & $N$ & N & $\mathrm{N}$ \\
\hline $\begin{array}{l}\text { Segment } \\
\text { https://github.com/Cardiac-MR-Group-Lund/ } \\
\text { segment-open/ }\end{array}$ & N & $\mathrm{N}$ & N (Windows-only) & Matlab only & N & Y & Y \\
\hline $\begin{array}{l}\text { MIA } \\
\text { http://mia.sourceforge.net/ }\end{array}$ & Y & N & N (POSIX-only) & $\mathrm{C}++$ only & $N$ & Y & Y \\
\hline $\begin{array}{l}\text { ITKSnap } \\
\text { http://www.itksnap.org/pmwiki/pmwiki.php }\end{array}$ & N & N & Y (binaries) & $\mathrm{C}++$ only & $N$ & Y & Y \\
\hline $\begin{array}{l}\text { 3DSlicer } \\
\text { https://www.slicer.org/ }\end{array}$ & $\mathrm{N}$ & $N$ & Y (binaries) & Python C++ & $\mathrm{N}$ & Y & Y \\
\hline $\begin{array}{l}\text { Osirix } \\
\text { http://www.osirix-viewer.com/ }\end{array}$ & $\mathrm{N}$ & N & N (Mac only) & $\begin{array}{l}\text { Objective C } \\
\text { only }\end{array}$ & N & Y & Y \\
\hline $\begin{array}{l}\text { Nora } \\
\text { http://www.nora-imaging.com/ }\end{array}$ & $\mathrm{N}$ & Y & Y & N & $\mathrm{N}$ & Y & $N$ \\
\hline $\begin{array}{l}\text { CoreSlicer } \\
\text { https://www.coreslicer.com }\end{array}$ & Y & Y & Y & $\begin{array}{l}\text { Any } \\
\text { language }\end{array}$ & Y & Y & Y \\
\hline
\end{tabular}




\section{Implementation}

\section{Specifications of CoreSlicer}

CoreSlicer is a browser-based application and is written entirely in Javascript, conformant to the ECMAScript 5 specification. A public running instance of CoreSlicer is provided free of use at www.coreslicer.com. This public running instance showcases elemental plugins for segmentation of abdominal structures (Additional files 1 and 2). Users may also download a standalone version of CoreSlicer free of charge at https://github.com/louismullie/coreslicer. The standalone version of CoreSlicer is released under the MIT license.

\section{Testing requirements}

CoreSlicer is developed for and was tested on Google Chrome, versions 65.0 and above. The Google Chrome platform was selected due to its widespread adoption and stability across multiple operating systems. Other browsers are not currently supported. Tests were conducted on a machine with a $2 \mathrm{GHz}$ intel Core i 5 processor and $8 \mathrm{~GB}$ of rapid-access memory (RAM). A minimum 1 GB of RAM is recommended to load full-body CT scans at $2.5 \mathrm{~mm}$ slice thickness, or $2 \mathrm{~GB}$ RAM at $1 \mathrm{~mm}$ slice thickness. A minimum processor speed of $1.0 \mathrm{GHz}$ is recommended to support interface drawing functions.

\section{Program structure}

CoreSlicer is divided into 4 main modules (Fig. 2). As is common for web applications, a "model-view-controller" architecture is employed to enforce separation of concerns between different application modules [20]. The "Uploader" controlled provides functionality for loading DICOM files from disk. The "Series" controlled handles selection of a series of interest within a DICOM file and decompression of the DICOM file, if necessary. The "Level" controlled provides functionality to select a level of interest on a sagittal reconstruction. The "Regions" controller provides functionality for displaying DICOM images and creating and editing regions of interest (ROIs).

\section{Graphical user interface}

The CoreSlicer interface is organized into 4 main windows (Fig. 3). From the "Uploader" window (Fig. 3a), users can load one or more DICOM file(s) by dragging and dropping or via the operating system's native file select input. From the "Series" window, users can select a

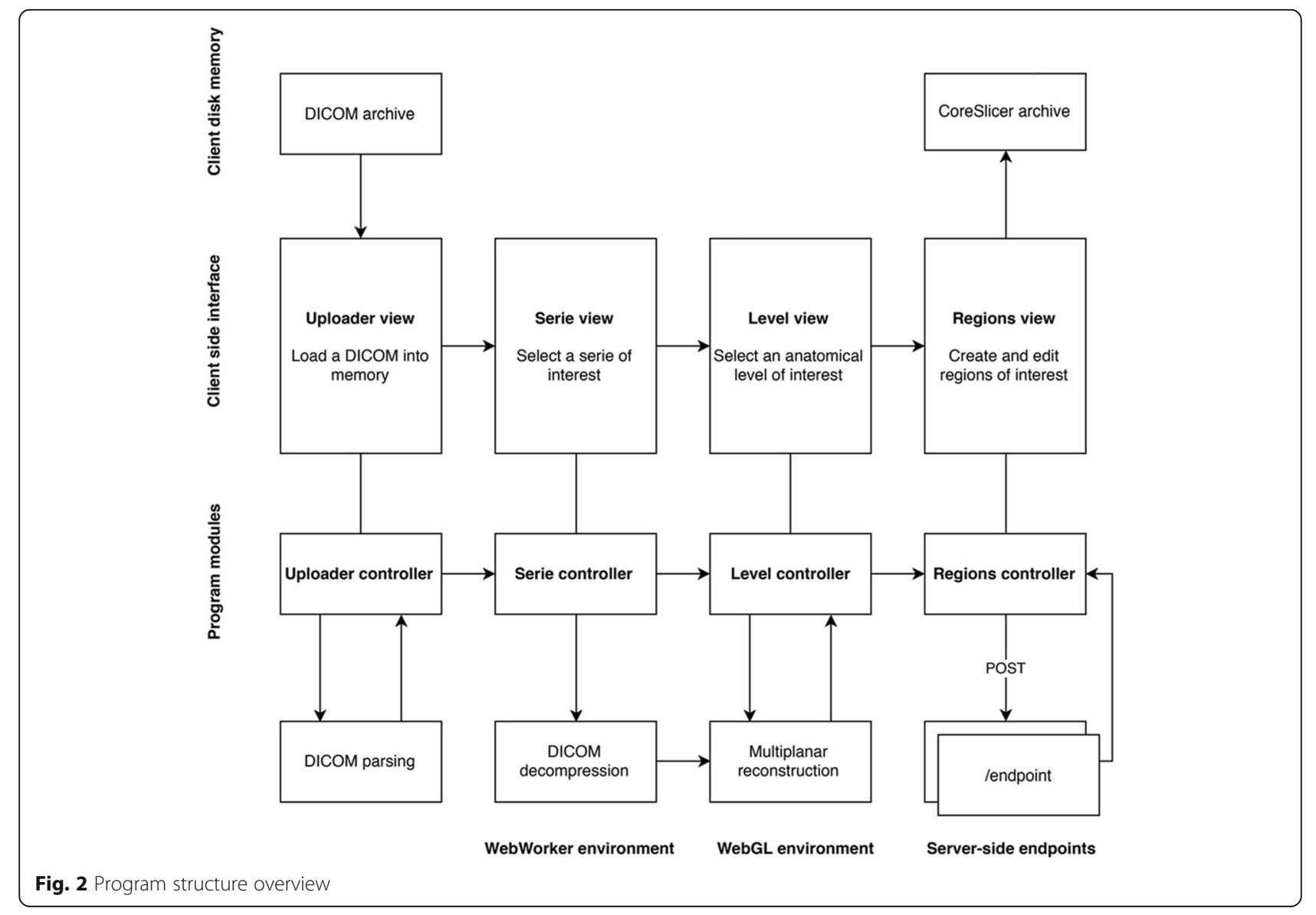


series of interest among those contained in the DICOM archive. From the "Level" window (Fig. 3b), users can select a level of interest on a sagittal reconstruction image of the previously selected series. From the "Region" window (Fig. 3c), users can define one or more ROIs, and assign a custom HTTP endpoint that provides automatic segmentation functionality for the relevant ROI. Users can draw and edit an ROI using the threshold brush and eraser tools. Results are exported to a ZIP archive containing the segmentation masks as well as area and Hounsfield unit measurements in comma-separated value (CSV) format.

\section{Extensibility via plugins}

CoreSlicer can be extended via user-supplied webhooks that provide segmentation functionality over an HTTP interface. CoreSlicer's plugin API structure is illustrated in Fig. 4.

From the "Regions" window, when creating a new custom region, CoreSlicer allows users to assign a custom
HTTP webhook that is triggered when a user initiates the draw action assigned to the corresponding ROI. In response, a POST request is sent to the assigned endpoint containing the anonymized DICOM image binary data and additional slice information encoded as a multipart FormData object (request MIME type multipart/ form-data). User endpoints return a binary segmentation mask in PNG format (response MIME type image/png), where all non-zero pixels are assigned a value of 1 . Further information is available from the user manual, which can be found on Github (https://github.com/ louismullie/coreslicer).

\section{Local deployment}

Deployment on a local server can be performed with minimal technical knowledge using Node.js, an open source programming runtime that is supported by all major operating systems including Windows, Mac OS and UNIX. Local deployments can be updated to the latest public version of CoreSlicer's code via a single

\section{a}

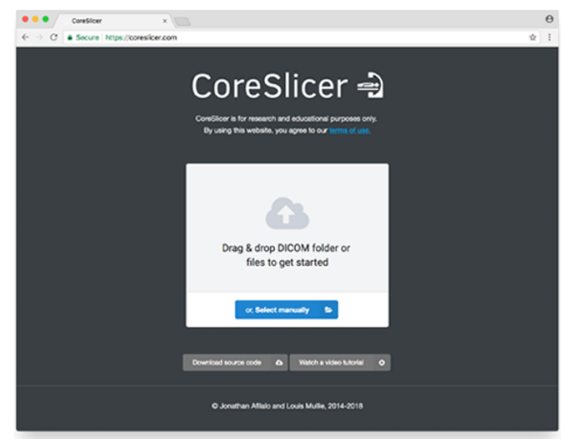

b

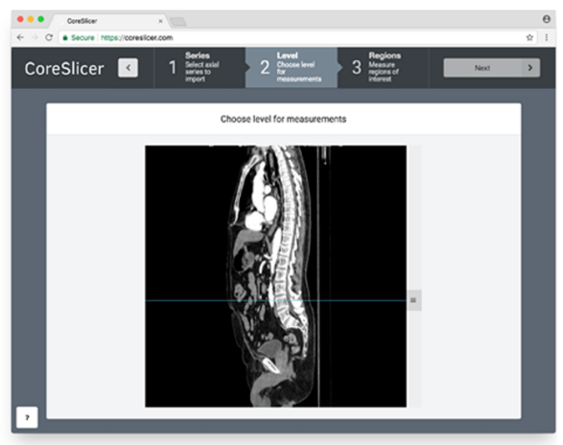

C

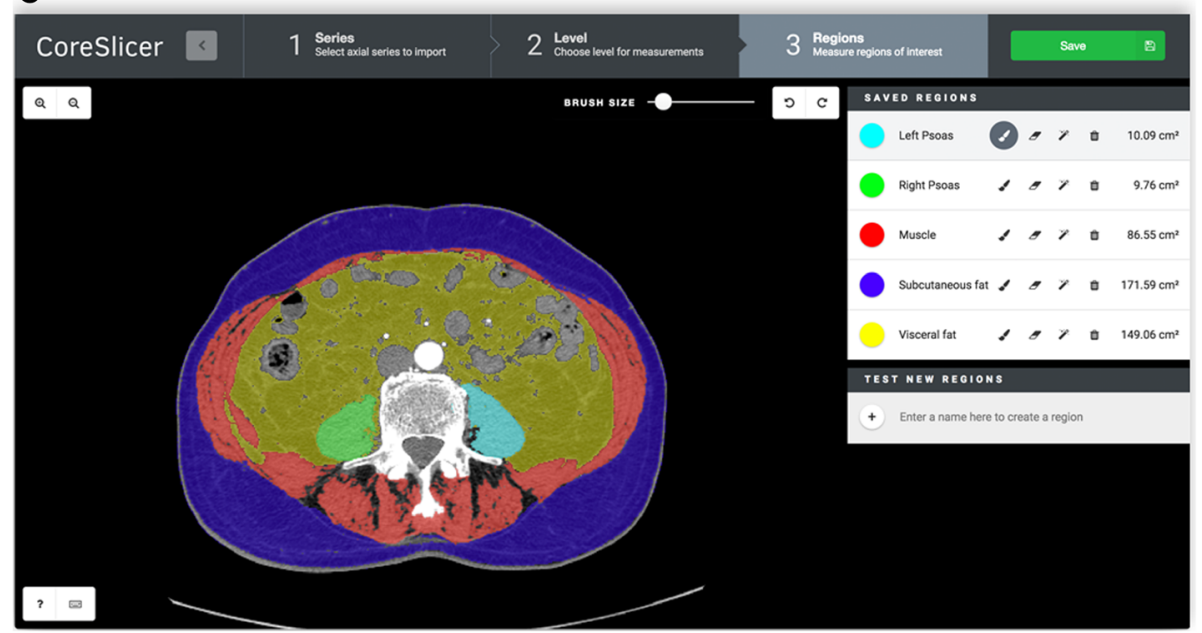

Fig. 3 Graphical user interface overview. Panel a shows the "Uploader" window, where DICOM archives can be imported. Panel b shows the "Level" window, using which an anatomical level can be selected. Panel c shows the "Region" window, using which regions of interest can be segmented 


\section{Regions of interest Plugin endpoints}

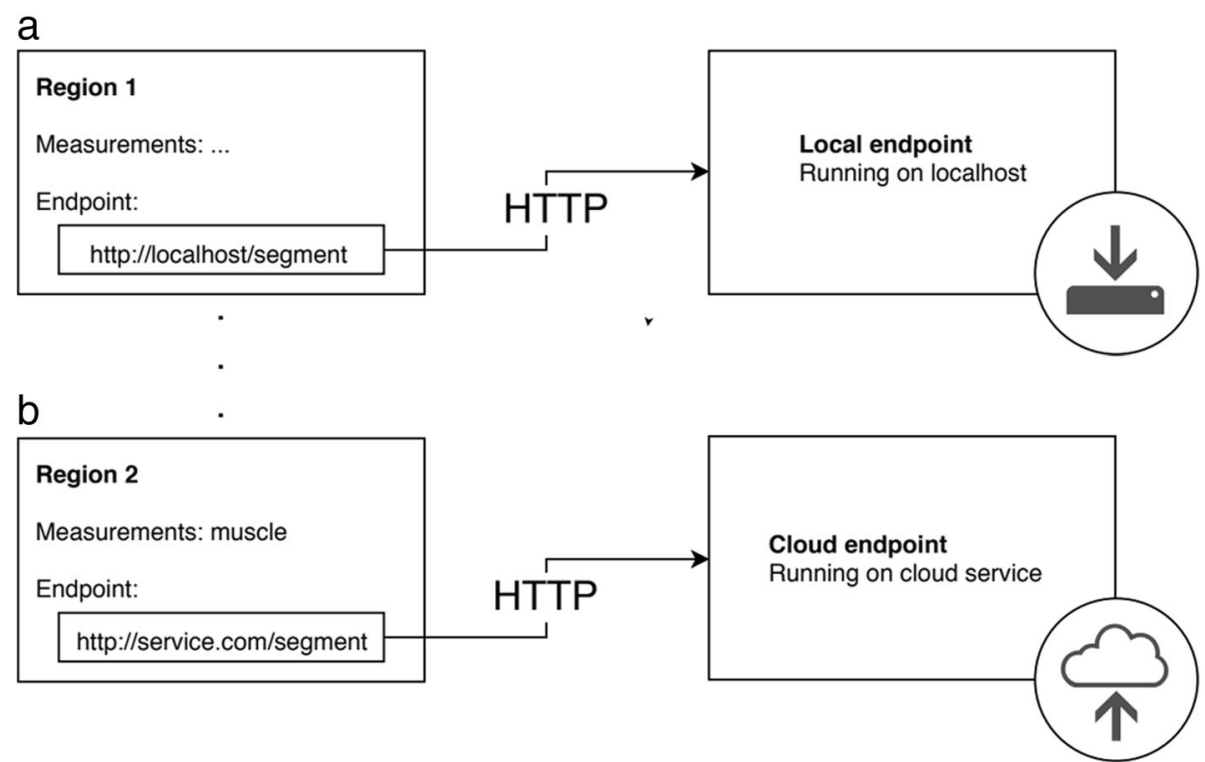

Fig. 4 Plugin architecture overview. Panel a shows an example of a plugin served on a local endpoint. Panel $\mathbf{b}$ shows an example of a plugin served on a remote endpoint

command, using the Git version management system. A detailed guide on how to install a local deployment and retrieve updates is available at https://github.com/louismullie/coreslicer.

\section{Implementation challenges}

Although highly flexible and granular, the DICOM standard is complex to manipulate because it supports multiple numerical representation, data sorting and compression formats. Cornerstone.js, an open-source DICOM utility library, was used to parse DICOM attributes and render DICOM images. A custom algorithm was implemented to reorder slices based on their position in space as determined from DICOM attributes. Jpx.js, an open-source JPX decompression implementation forked from Mozilla's PDF.js project, was used to provide support for decompression of DICOM archives.

While browser-based applications are highly advantageous for cross-platform compatibility, they represent a "resource-costly" environment in which large file manipulation are memory-expensive operations. The embarrassingly parallel nature of DICOM slice decompression enabled the use of concurrency to increase processing speed by distributing data to a pool of 8 WebWorker processes running simultaneously. To further optimize performance, CoreSlicer adheres to the "lazy loading" design pattern, deferring the initialization of memory-costly data structures until the point at which the data is needed for user interface rendering.
In addition to performance limitations with regards to manipulation of large files, browser environments have limited native abilities for complex numerical operations. To provide fast multiplanar reconstruction (MPR) for CoreSlicer, we implemented an MPR algorithm using WebGL, an HTML5 standard that allows for accelerated manipulation of 3-dimensional data on the graphics processing unit (GPU). To store DICOM images in memory and manipulate them, CoreSlicer leverages TypedArray objects, which provide a fast mechanism for accessing raw binary data in Javascript, and which can be easily transferred to the GPU using WebGL. The combination of these strategies allows CoreSlicer to efficiently reslice full-body CT scans, with minimal computational time, on the order of $<500 \mathrm{~ms}$.

To enable smooth drawing operations for manual labeling of DICOM images, CoreSlicer leverages Canvas API, which enables hardware-accelerated rendering of two-dimensional graphics in the browser. A system of stacked Canvas objects is used to represent multiple overlying layers in transparency. Tegaki.js, an open-source HTML drawing library, is employed as a foundation for CoreSlicer's customized DICOM drawing tools.

\section{Security and privacy}

Ensuring the security and privacy of user data is a key implementation challenge in any web environment. The core application functionality of CoreSlicer is implemented entirely on the client machine and does not 
require any transmission of data. This includes loading DICOM files, decompressing image files if needed, performing multiplanar reconstruction, as well as manually drawing and editing measurements. The use of optional segmentation plugins requires transmission of information over the Internet. This information is protected in transit using RSA encryption with 4096-bit keys, via the Secure Sockets Layer (SSL) protocol. In addition, through its plugin interface, CoreSlicer allows users to securely transmit data directly from their machine to user-controlled processing endpoints, without any information transiting via CoreSlicer's servers. Users who desire enhanced privacy can run the CoreSlicer interface on their local machines. CoreSlicer automatically strips DICOM files of identifying information prior to any form of external transmission in order to prevent disclosure of protected health information.

\section{Validation methods}

Measurements from CoreSlicer were validated against slice-O-matic, a commercial cadaver-validated reference software. Cross-sectional area measurements (Fig. 4) were performed by two trained observers (LM and JA), respectively designated observer $\mathrm{A}$ and observer $\mathrm{B}$. Observer A performed measurements of VFA, SFA, TLMA and PMA in triplicate, once using the slice-O-matic software package (version 5.0), and twice using the manual drawing tools in the CoreSlicer web interface (version 1.0). Repeated measurements were performed at least 1 week apart to limit observer bias. In a subset of 20 scans, observers A and B each obtained measurements of VFA, SFA, TLMA and PMA using CoreSlicer. All measurements were performed on axial series at the superior aspect of L4, immediately below the vertebral endplate.

ROIs were defined as: psoas muscle area (PMA), total lumbar muscle area (TLMA), visceral fat area (VFA) and subcutaneous fat area (SFA). These were chosen among markers illustrated in Table 1 based on the robustness of the supporting clinical evidence. A representative labeled image is illustrated in Fig. 5. Hounsfield unit ranges were -190 to -30 for adipose tissue (VFA and SFA), and -29 to 150 for skeletal muscle (TLMA and PMA) [9].

\section{Statistical analyses}

For comparisons between sets of measurements, mean difference, 95\% level of agreement (LOA), and Spearman's correlation coefficient were calculated. Results are presented using Bland-Altman plots (Additional file 3). Additionally, for the main outcome of interest, which compared measurements in CoreSlicer with measurements in slice-O-matic, the intraclass correlation coefficient was calculated, and power analysis was performed. Assuming a hypothesized ICC of 0.99 , and a null ICC value of 0.90 , a sample of 50 subjects ( 2 measurements per subject) has $100 \%$ power to detect a difference between the two measurement methods at a 5\% type 1 error rate. All statistical analyses were performed with the STATA software package (version 14.0, College Station, Texas).

\section{Results}

A total of $50 \mathrm{CT}$ scans were drawn and analyzed from a cohort of older adults undergoing a routine preoperative $\mathrm{CT}$ scans during assessment for a heart valve procedure. The population comprised 24 women and 26 men, with a mean age of 80 years (range 64 to 96 years). The mean BMI was 26.2 (range 20.5 to 44.5). Descriptive statistics of the study population are presented in Table 4.

Mean cross-sectional areas were $202.6 \pm 82.5 \mathrm{~cm}^{2}$ for VFA, $214.2 \pm 105.8 \mathrm{~cm}^{2}$ for SFA, $119.05 \pm 26.5 \mathrm{~cm}^{2}$ for TLMA, and $19.77 \pm 5.6 \mathrm{~cm}^{2}$ for PMA. In Fig. 6, a

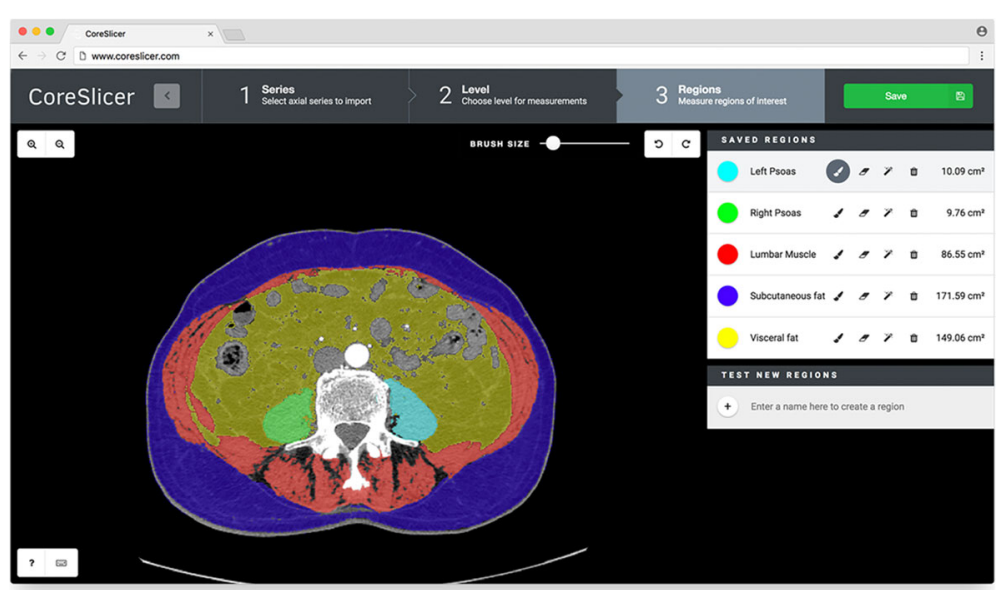

Fig. 5 Illustration of muscle and fat segmentation at L4 in CoreSlicer 
Table 4 Descriptive statistics of the study population

\begin{tabular}{lll}
\hline Variable & Males $(N=26)$ & Females $(N=24)$ \\
\hline Age $(\mathrm{y})$ & $81.4 \pm 7.6(64-96)$ & $79.8 \pm 7(67-92)$ \\
Height $(\mathrm{m})$ & $1.7 \pm 0.1(1.6-1.9)$ & $1.6 \pm 0.1(1.5-1.8)$ \\
Weight $(\mathrm{kg})$ & $75.7 \pm 10.4(54.5-100)$ & $67.4 \pm 15.3(48.0-99.0)$ \\
BMI $\left(\mathrm{kg} / \mathrm{m}^{2}\right)$ & $26.1 \pm 2.8(20.2-34.2)$ & $26.2 \pm 5.8(21.5-44.5)$ \\
VFA $\left(\mathrm{cm}^{2}\right)$ & $252.5 \pm 122.7(114-603.9)$ & $252.5 \pm 122.7(114-603.9)$ \\
SFA $\left(\mathrm{cm}^{2}\right)$ & $198.9 \pm 62.9(113.3-368.3)$ & $208.6 \pm 101.9(89.4-584.6)$ \\
TLMA $\left(\mathrm{cm}^{2}\right)$ & $134.5 \pm 22.3(87.1-173.4)$ & $102.7 \pm 20.8(80.0-155.7)$ \\
\hline
\end{tabular}

Bland-Altman analysis is presented for comparison of cross-sectional area measurement differences between CoreSlicer and Slice-O-Matic. Mean absolute differences in cross-sectional areas were $4.2 \mathrm{~cm}^{2}\left(-4.7\right.$ to $\left.13.1 \mathrm{~cm}^{2}\right)$ for VFA, $4.8 \mathrm{~cm}^{2}\left(-7.7\right.$ to $\left.14.7 \mathrm{~cm}^{2}\right)$ for SFA, $-3.8 \mathrm{~cm}^{2}$ $\left(-10.1\right.$ to $\left.-2.4 \mathrm{~cm}^{2}\right)$ for TLMA, and $0.4 \mathrm{~cm}^{2}(-1.4$ to $2.2 \mathrm{~cm}^{2}$ ) for PMA. Mean differences in cross-sectional areas expressed as relative percentages were $2.1 \%(-2.4$ to $6.5 \%$ ) for VFA, $2.3 \%$ (-3.9 to $7.3 \%$ ) for SFA, $-3.0 \%$ (- 7.9 to $1.9 \%$ ) for TLMA and $2.0 \%$ (- 7.0 to $11.0 \%$ ) for PMA. Intra-class correlation coefficients were 0.998 for VFA, 0.999 for SFA, 0.984 for TMA, 0.994 for PMA. Spearman's correlation coefficient exceeded 0.99 for all ROIs, with $p$ values of $<0.001$.

In Figs. 7 and 8, Bland-Altman analyses are shown for intra- and inter-observer measurements in CoreSlicer. For repeated manual measurements by observer A, mean absolute differences in cross-sectional areas were
$-0.2 \mathrm{~cm}^{2}$ ( -6.0 to $5.8 \mathrm{~cm}^{2}$ ) for VFA, $-1.1 \mathrm{~cm}^{2}$ (- 8.0 to $\left.5.8 \mathrm{~cm}^{2}\right)$ for SFA, $0.4 \mathrm{~cm}^{2}\left(-1.7\right.$ to $\left.2.4 \mathrm{~cm}^{2}\right)$ for TLMA, and $0.6 \mathrm{~cm}^{2}$ (- 5.7 to $\left.7.0 \mathrm{~cm}^{2}\right)$ for PMA. For comparison of automated measurements plus manual corrections between observers $\mathrm{A}$ and $\mathrm{B}$, mean differences in cross-sectional areas were $1.2 \mathrm{~cm}^{2}\left(-2.7\right.$ to $\left.5.6 \mathrm{~cm}^{2}\right)$ for VFA, $-0.2 \mathrm{~cm}^{2}$ (- 4.4 to $4.0 \mathrm{~cm}^{2}$ for SFA), $-0.8 \mathrm{~cm}^{2}(-5.2$ to $\left.3.7 \mathrm{~cm}^{2}\right)$ for TLMA, and $-0.5 \mathrm{~cm}^{2}\left(-0.9\right.$ to $\left.1.8 \mathrm{~cm}^{2}\right)$ for PMA.

\section{Discussion}

In this study, we introduced the CoreSlicer web interface and software toolkit for analytic morphomics on CT scan images. CoreSlicer is the first open-source web-based MIA designed and optimized specifically for analytic morphomics. Given its implementation as a browser application, CoreSlicer is portable across all major operating systems (Windows, MacOS, Linux and Chrome OS), addressing an important limitation of several existing tools and facilitating collaboration between researchers working on different platforms. A core set of tools required to manually review and correct segmentation results is provided within the interface, and the workflow is organized in a simple to use, step-by-step wizard. The average time required in CoreSlicer for measurement of psoas muscle area, one of the most robustly validated morphomic markers, was $22 \pm 3 \mathrm{~s}$, supporting the benefit of a streamlined, workfloworiented interface.

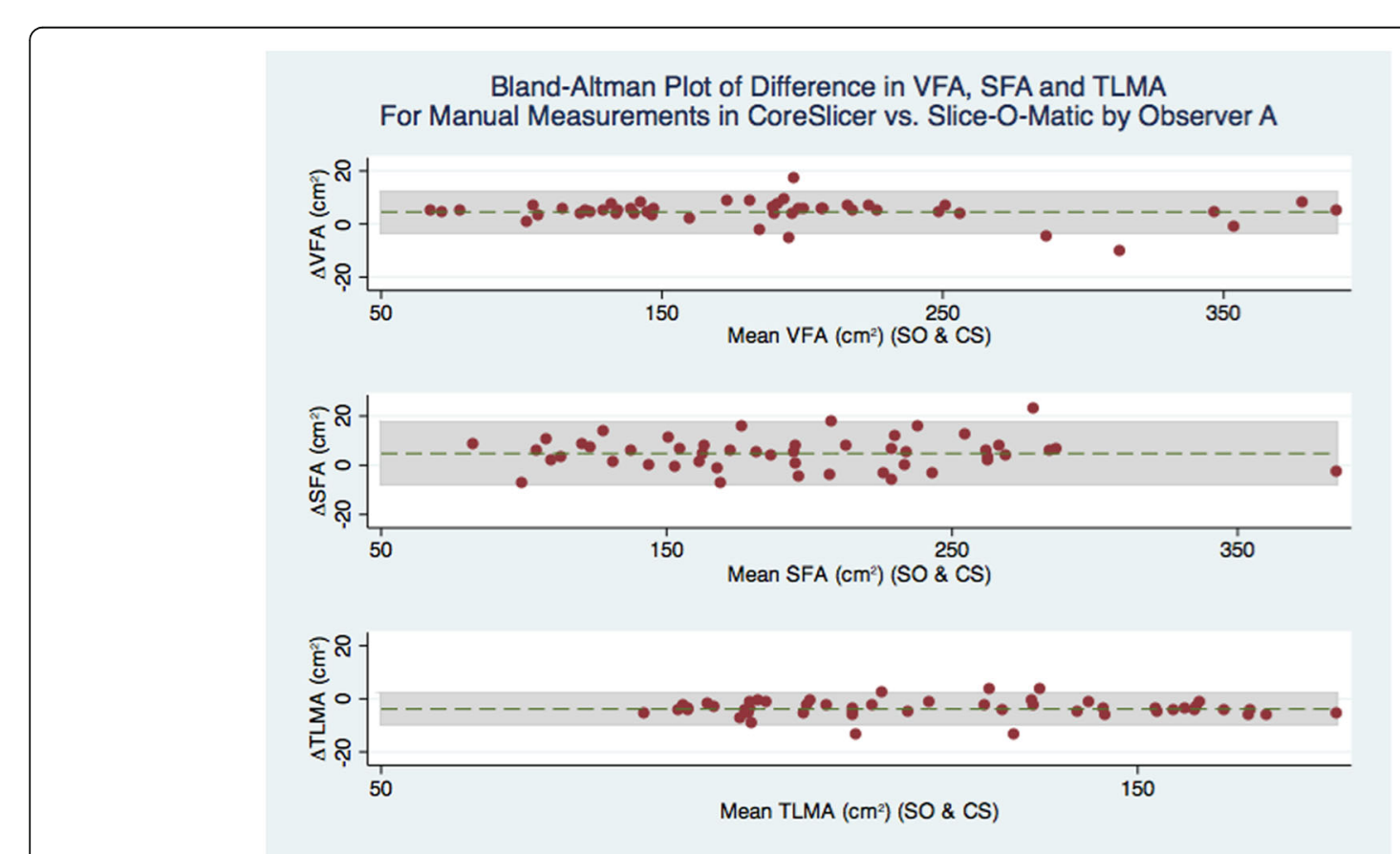

Fig. 6 Bland-Altman plot of difference in VFA, SFA and TLMA for manual measurements in CoreSlicer vs. Slice-O-Matic by Observer A 
Bland-Altman Plot of Difference in VFA, SFA and TLMA

For Repeated Manual Measurements in CoreSlicer by Observer A
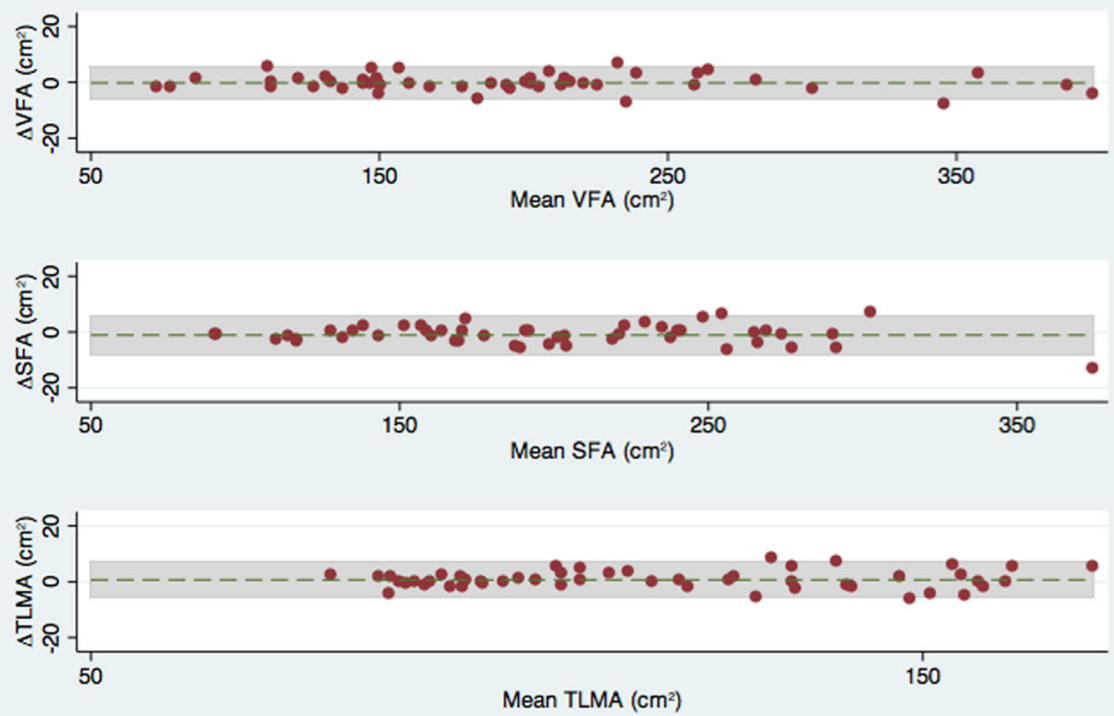

Fig. 7 Bland-Altman plot of difference in VFA, SFA and TLMA for repeated manual measurements in CoreSlicer by Observer A

Implementation challenges were addressed with state of the art browser application technologies including distributed processing of large files using WebWorkers, hardware-accelerated 2D drawing using the Canvas object, 3D multiplanar reconstruction on the GPU using WebGL, and memory optimizations using TypedArrays.

Researchers can use CoreSlicer to rapidly prototype image segmentation tools in the language of their choice using a standardized HTTP interface. Additionally,

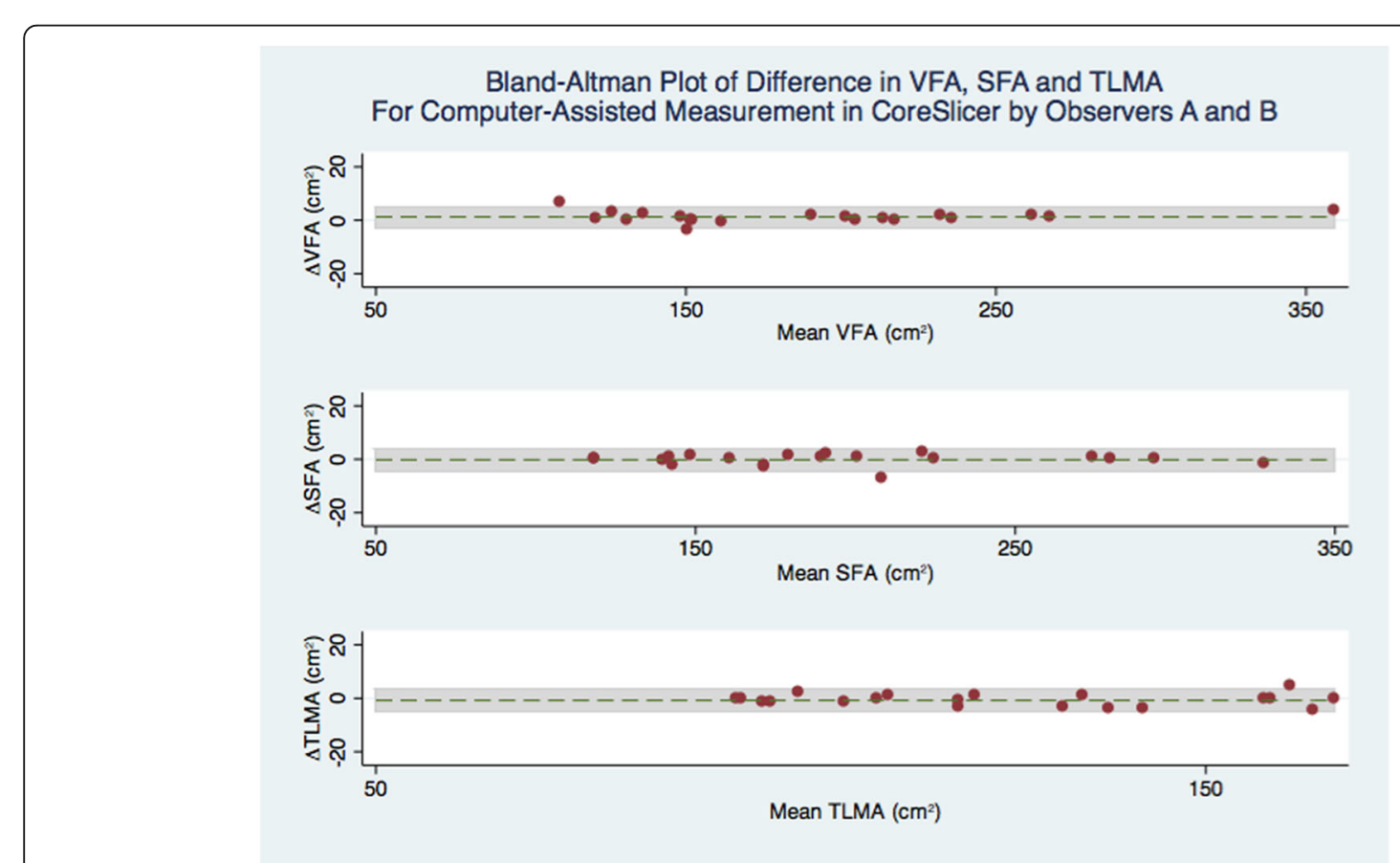

Fig. 8 Bland-Altman plot of difference in VFA, SFA and TLMA for computed-assisted measurements in CoreSlicer by Observers A and B 
CoreSlicer is the first web-based MIA software with out-of-the-box support for asynchronous plugin execution, enabling the use of segmentation tools served in the cloud at the click of a button. For researchers seeking to expand, refine, or adapt the capabilities CoreSlicer, the source code has been made available via an open source repository.

We conducted duplicate measurements of wellvalidated fat and muscle morphomic markers using CoreSlicer and slice-O-matic, and demonstrated good agreement between the two methods. The limits of agreement were comparable with previously reported values for comparison of the NIH Image and Slice-O-Matic software packages [8]. The intra- and inter-observer variability of measurements was also comparable with previously reported results [21], with the relative differences all being $<3 \%$ and unlikely to be clinically significant. Thus, measurements of VFA, SFA and TLMA in CoreSlicer were reproducible and accurate in relation to reference software.

The potential impact of CoreSlicer as a translational tool for morphomics has been demonstrated in three studies aiming to validate the prognostic value of PMA in cardiac and vascular surgery patients. Our work and that of others has shown that psoas muscle area (PMA), a surrogate of lean muscle mass and lower extremity strength, correlates with clinical frailty scores and is an important risk factor for morbidity and mortality following invasive procedures (Table 1). Mamane et al. [32] showed that PMA measured using CoreSlicer was predictive of mortality in elderly women undergoing TAVR. Drudi et al. [38] similarly showed that PMA was predictive of mortality in patients undergoing abdominal aortic aneurysm repair. Zuckerman et al. [30] used CoreSlicer to show that PMA correlated with length of stay following major cardiac surgery. Thus, measurements of PMA in CoreSlicer were incrementally predictive of adverse health outcomes in vulnerable patients undergoing invasive procedures. These measurements could be obtained by medical trainees with no prior medical image analysis training in under $1 \mathrm{~min}$.

These examples illustrate how morphomics can empower healthcare professionals with prognostic information to tailor treatment strategies and individualize care. Patients found to have low muscle mass, often unrecognized by the de facto "eyeball assessment", may benefit from targeted interventions such as exercise therapy and nutritional supplementation to build muscle mass and strength and minimize their risk of failed recovery after an illness or surgery [22]. This information, which has been consciously absent from the clinical arena owing to the inaccessible nature of its measurement tools, is now readily obtainable within $<1 \mathrm{~min}$ at the point of care by healthcare professionals with minimal pre-training.

\section{Limitations}

The results of this study must be considered in light of the following limitations. First, since CT incurs ionizing radiation and is not appropriate for the sole purpose of body composition analysis, CoreSlicer is currently limited to the analysis of images that have been acquired for clinical purposes. Protocols are in development to acquire limited slices with lower radiation. Second, although the CoreSlicer software has been designed to visualize DICOM images produced by both CT and MRI scanners, only the former modality was tested and validated in this initial release of the software. Given the widespread availability of clinically indicated CT studies, we believe that this in no way limits the potential large-scale impact of our software. Third, CoreSlicer is currently capable of performing measurements on one slice per study and is therefore not amenable to compute volumetric measurements in its current iteration. Importantly, the aims of the CoreSlicer project are not to provide a comprehensive MIA or image segmentation toolkit, but rather to provide a streamlined workflow for analytic morphomics, and to maintain the simplicity to achieve our translational goal of a clinician-friendly interface. A large number of single- slice morphomic markers have already been validated and can be measured using CoreSlicer. Once careful planning identifies how to optimally integrate multi-slice functionality while keeping the interface complexity at a minimum, three-dimensional visualization will be implemented using existing open-source libraries [23].

\section{Conclusions}

CoreSlicer is a free and open-source web-based interface aiming to facilitate measurement of analytic morphomics on DICOM images by non-technically skilled clinicians. CoreSlicer features a full set of DICOM viewing tools and extensible plugin interface to facilitate rapid prototyping and validation of new morphomic markers by researchers. In this study, the CoreSlicer interface and functionality is described, and validity of CoreSlicer measurements is established by comparing its results with reference software on a set of 50 abdominal CT scans, demonstrating good reproducibility and agreement with reference software. We present published studies illustrating the clinical relevance of morphomic measurements obtained in CoreSlicer in three distinct populations of cardiovascular patients.

\section{Availability and requirements \\ Project name: CoreSlicer. \\ Project home page: www.coreslicer.com \\ Operating system(s): platform independent. \\ Programming language: Javascript. \\ Other requirements: none. \\ License: MIT.}

Restrictions: CoreSlicer is not licensed for clinical or commercial use. 


\section{Additional files}

Additional file 1: Algorithm for computer-assisted adipose and muscle tissue segmentation on abdominal $\subset$ scan images. Describes the elemental segmentation algorithms used to showcase CoreSlicer's functionality on www.coreslicer.com. (DOCX $100 \mathrm{~kb}$ )

Additional file 2: Figure S1. Illustration of muscle and adipose tissue boundary detection in pseudo-polar coordinates. (PDF 1579 kb)

Additional file 3: Figure S2. Bland-Altman plot of difference in VFA, SFA and TLMA for manual measurements in CoreSlicer by Observers A versus automated segmentation. (PDF $836 \mathrm{~kb}$ )

\section{Abbreviations}

BMI: Body mass index; CT: Computed tomography; DICOM: Digital Imaging and Communications in Medicine; GPU: Graphics processing unit; MIA : Medical image analysis; MR: Magnetic resonance; PMA: Psoas muscle area; ROI: Region of interest; SFA: Subcutaneous adipose tissue area; TLMA: Total skeletal muscle area; VFA: Visceral adipose tissue area

\section{Acknowledgements}

We would like to acknowledge Christophe Marois for his help in developing the Coreslicer user interface.

Trademarks: DICOM is a registered trademark of the National Electrical Manufacturers Association for its standards publications relating to digital communication of medical information.

\section{Funding}

Jonathan Afilalo is supported by the Fonds de recherche du Québec en Santé, Heart and Stroke Foundation of Canada, and Canadian Institutes of Health Research.

\section{Availability of data and materials}

The anonymized datasets used in the current study are available from the corresponding author upon reasonable request.

\section{Authors' contributions}

LM designed and programmed the software and wrote major portions of the paper. JA oversaw writing the software and wrote and edited portions the paper. LM and JA collected measurements. Both authors read and approved the final manuscript.

\section{Ethics approval and consent to participate}

The study protocol was approved by the Ethics Review Board of the Jewish General Hospital.

\section{Consent for publication}

Not applicable.

\section{Competing interests}

Louis Mullie and Jonathan Afilalo declare that they have no conflicts of interest. Louis Mullie and Jonathan Afilalo developed the CoreSlicer software but do not hold any financial incentives or patents related to its use.

\section{Publisher's Note}

Springer Nature remains neutral with regard to jurisdictional claims in published maps and institutional affiliations.

\section{Author details}

'Department of Medicine, McGill University, 3755 Cote Ste Catherine Rd, E-222, Montreal, QC H3T 1E2, Canada. ²Division of Cardiology, McGill University, Montreal, QC, Canada. ${ }^{3}$ Centre for Clinical Epidemiology, Lady Davis Institute, Jewish General Hospital, McGill University, Montreal, QC, Canada.
Received: 24 April 2018 Accepted: 21 January 2019

Published online: 11 February 2019

\section{References}

1. Friedman J, Lussiez A, Sullivan J, Wang S, Englesbe M. Implications of sarcopenia in major surgery. Nutr Clin Pract. 2015 Apr;30(2):175-9.

2. Stidham RW, Waljee AK, Day NM, Bergmans CL, Zahn KM, Higgins PD, et al. Body fat composition assessment using analytic morphomics predicts infectious complications after bowel resection in Crohn's disease. Inflamm Bowel Dis. 2015 Jun;21(6):1306-13.

3. Chughtai K, Song Y, Zhang P, Derstine B, Gatza E, Friedman J, et al. Analytic morphomics: a novel CT imaging approach to quantify adipose tissue and muscle composition in allogeneic hematopoietic cell transplantation. Bone Marrow Transplant. 2016 Mar;51(3):446-50.

4. Singal AG, Zhang P, Waljee AK, Ananthakrishnan L, Parikh ND, Sharma P, et al. Body composition features predict overall survival in patients with hepatocellular carcinoma. Clin Transl Gastroenterol. 2016 May 26;7:e172.

5. Morphomics Research Group. Michigan Medicine. http://www.med.umich edu/surgery/morphomics/index.html. 2018. Accessed 24 Apr 2018.

6. Smith-Bindman R, Miglioretti DL, Johnson E, Lee C, Feigelson HS, Flynn M, et al. Use of diagnostic imaging studies and associated radiation exposure for patients enrolled in large integrated health care systems, 1996-2010. JAMA. 2012;307(22):2400-9.

7. C Anastasopoulos, M Reisert, E Kellner. "Nora Imaging": A Web-Based Platform for Medical Imaging. Neuropediatrics. 2017; doi: https://doi.org/10. 1055/s-0037-1602977

8. Schneider CA, Rasband WS, Eliceiri KW. NIH image to ImageJ: 25 years of image analysis. Nat Methods. 2012;9(7):671-5.

9. Abate N, Burns D, Peshock RM, Garg A, Grundy SM. Estimation of adipose tissue mass by magnetic resonance imaging: validation against dissection in human cadavers. J Lipid Res. 1994:35(8):1490-6.

10. Martijn van W, Hallvard T. Monitor Changes: Interaction Patterns in User Interfaces: 2000; doi: 10.1.1.36.7484.

11. Wollny G, Kellman P, Ledesma-Carbayo MJ, Skinner MM, Hublin JJ, Hierl T. MIA - a free and open source software for gray scale medical image analysis. Source Code Biol Med. 2013;8(1):20.

12. Gelaude F, Vander Sloten J, Lauwers B. Accuracy assessment of CT-based outer surface femur meshes. Comput Aided Surg. 2008;13(4):188-99.

13. Heiberg E, Sjögren J, Ugander M, Carlsson M, Engblom $H$, Arheden $H$. Design and validation of segment--freely available software for cardiovascular image analysis. BMC Med Imaging. 2010;10:1.

14. Rosset A, Spadola L, Ratib O. OsiriX: an open-source software for navigating in multidimensional DICOM images. J Digit Imaging. 2004;17(3):205-16.

15. Yushkevich PA, Piven J, Hazlett HC, Smith RG, Ho S, Gee JC, et al. Userguided $3 \mathrm{D}$ active contour segmentation of anatomical structures: significantly improved efficiency and reliability. Neurolmage. 2006:31(3): 1116-28.

16. Pieper S, Halle M, Kikinis R. 3D Slicer. IEEE International Symposium on Biomedical Imaging: Nano to Macro 2004; doi: https://doi.org/10.1109/ISBI. 2004.1398617

17. Susumu M, Dan W, Can C. MRICloud: delivering high-throughput MRI Neuroinformatics as cloud-based software as a service. Computing in Science \& Engineering. 2016;18(5):21-35

18. Sherif T, Rioux P, Rousseau ME, Kassis N, Beck N, Adalat R, et al. CBRAIN: a web-based, distributed computing platform for collaborative neuroimaging research. Front Neuroinform. 2014;8:54.

19. Manjón JV, Coupé P. volBrain: An Online MRI Brain Volumetry System. Front Neuroinform. 2016;10:30

20. A Leff, JT Rayfield. Web-application development using the Model/Niew/ Controller design pattern Enterprise Distributed Object Computing Conference: 2001; doi: https://doi.org/10.1109/EDOC.2001.950428.

21. Sottier D, Petit JM, Guiu S, Hamza S, Benhamiche H, Hillon P, et al. Quantification of the visceral and subcutaneous fat by computed tomography: interobserver correlation of a single slice technique. Diagn Interv Imaging. 2013;94(9):879-84.

22. Malafarina V, Uriz-Otano F, Iniesta R, Gil-Guerrero L. Effectiveness of nutritional supplementation on muscle mass in treatment of sarcopenia in old age: a systematic review. J Am Med Dir Assoc. 2013;14(1):10-7.

23. Haehn D, Rannou N, Ahtam B, Grant E, Pienaar R. Neuroimaging in the browser using the $X$ toolkit. Neuroinformatics. 2014. https://doi.org/10.3389/ conf.fninf.2014.08.00101. 
24. Mitsiopoulos N, Baumgartner RN, Heymsfield SB, Lyons W, Gallagher D, Ross R. Cadaver validation of skeletal muscle measurement by magnetic resonance imaging and computerized tomography. J Appl Physiol (1985). 1998 Jul;:85(1):115-22.

25. Zakaria HM, Massie L, Basheer A, Elibe E, Boyce-Fappiano D, Shultz L, et al. Application of Morphometrics as a Predictor for Survival in Patients with Prostate Cancer Metastasis to the Spine. World Neurosurg. 2018.

26. Zakaria HM, Massie L, Basheer A, Boyce-Fappiano D, Elibe E, Schultz L, et al. Application of morphometrics as a predictor for survival in female patients with breast cancer spinal metastasis: a retrospective cohort study. Spine J. 2018.

27. Tsutsumi S, Kawahara T, Teranishi JI, Yao M, Uemura H. A low psoas muscle volume predicts longer hospitalization and cancer recurrence in upper urinary tract urothelial carcinoma. Mol Clin Oncol. 2018;8(2):320-2.

28. Indrakusuma R, Zijlmans $\mathrm{J}$, Jalalzadeh $H$, Planken RN, Balm R, Koelemay MJW. Psoas muscle area as a prognostic factor for survival in patients with an asymptomatic Infrarenal abdominal aortic aneurysm: a retrospective cohort study. Eur J Vasc Endovasc Surg. 2018;55(1):83-91.

29. Garg L, Agrawal S, Pew T, Hanzel GS, Abbas AE, Gallagher MJ, et al. Psoas muscle area as a predictor of outcomes in Transcatheter aortic valve implantation. Am J Cardiol. 2017;119(3):457-60.

30. Zuckerman J, Ades M, Mullie L, Trnkus A, Morin JF, Langlois Y, et al. Psoas muscle area and length of stay in older adults undergoing cardiac operations. Ann Thorac Surg. 2017;103(5):1498-504.

31. Miller BS, Ignatoski KM, Daignault S, Lindland C, Doherty M, Gauger PG, et al. Worsening central sarcopenia and increasing intra-abdominal fat correlate with decreased survival in patients with adrenocortical carcinoma. World J Surg. 2012;36(7):1509-16.

32. Mamane S, Mullie L, Piazza N, Martucci G, Morais J, Vigano A, et al. Psoas muscle area and all-cause mortality after Transcatheter aortic valve replacement: the Montreal-Munich study. Can J Cardiol. 2016;32(2):177-82.

33. Peng $P$, Hyder $O$, Firoozmand A, Kneuertz P, Schulick RD, Huang D, et al Impact of sarcopenia on outcomes following resection of pancreatic adenocarcinoma. J Gastrointest Surg. 2012;16(8):1478-86.

34. Krell RW, Kaul DR, Martin AR, Englesbe MJ, Sonnenday CJ, Cai S, et al. Association between sarcopenia and the risk of serious infection among adults undergoing liver transplantation. Liver Transpl. 2013;19(12):1396-402.

35. Smith AB, Deal AM, Yu H, Boyd B, Matthews J, Wallen EM, et al. Sarcopenia as a predictor of complications and survival following radical cystectomy. J Urol. 2014;191(6):1714-20.

36. Kuroki LM, Mangano M, Allsworth JE, Menias CO, Massad LS, Powell MA, et al. Pre-operative assessment of muscle mass to predict surgical complications and prognosis in patients with endometrial cancer. Ann Surg Oncol. 2015;22(3):972-9.

37. Wakabayashi H, Matsushima M, Uwano R, Watanabe N, Oritsu H, Shimizu Y. Skeletal muscle mass is associated with severe dysphagia in cancer patients. J Cachexia Sarcopenia Muscle. 2015;6(4):351-7.

38. Drudi LM, Phung K, Ades M, Zuckerman J, Mullie L, Steinmetz OK, et al. Psoas muscle area predicts all-cause mortality after endovascular and open aortic aneurysm repair. Eur J Vasc Endovasc Surg. 2016;52(6):764-9.

39. Peng PD, van Vledder MG, Tsai S, de Jong MC, Makary M, Ng J, et al. Sarcopenia negatively impacts short-term outcomes in patients undergoing hepatic resection for colorectal liver metastasis. HPB (Oxford). 2011:13(7):439-46.

40. Lee JS, He K, Harbaugh CM, Schaubel DE, Sonnenday CJ, Wang SC, et al. Frailty, core muscle size, and mortality in patients undergoing open abdominal aortic aneurysm repair. J Vasc Surg. 2011;53(4):912-7.

41. Miller AL, Englesbe MJ, Diehl KM, Chan CL, Cron DC, Derstine BA, et al. Preoperative psoas muscle size predicts postoperative delirium in older adults undergoing surgery: a pilot cohort study. J Am Geriatr Soc. 2017;65(1):e23-4.

42. Paknikar R, Friedman J, Cron D, Deeb GM, Chetcuti S, Grossman PM, et al. Psoas muscle size as a frailty measure for open and transcatheter aortic valve replacement. J Thorac Cardiovasc Surg. 2016;151(3):745-51.

43. Sheetz KH, Zhao L, Holcombe SA, Wang SC, Reddy RM, Lin J, et al. Decreased core muscle size is associated with worse patient survival following esophagectomy for cancer. Dis Esophagus. 2013;26(7):716-22.

44. Underwood PW, Cron DC, Terjimanian MN, Wang SC, Englesbe MJ, Waits SA. Sarcopenia and failure to rescue following liver transplantation. Clin Transpl. 2015;29(12):1076-80.

45. Caram MV, Bellile EL, Englesbe MJ, Terjimanian M, Wang SC, Griggs JJ, et al. Sarcopenia is associated with autologous transplant-related outcomes in patients with lymphoma. Leuk Lymphoma. 2015;56(10):2855-62.
46. Sabel MS, Lee J, Cai S, Englesbe MJ, Holcombe S, Wang S. Sarcopenia as a prognostic factor among patients with stage III melanoma. Ann Surg Oncol. 2011;18(13):3579-85.

47. Hawkins RB, Mehaffey JH, Charles EJ, Kern JA, Lim DS, Teman NR, et al. Psoas Muscle Size Predicts Risk-Adjusted Outcomes After Surgical Aortic Valve Replacement. Ann Thorac Surg. 2018.

48. Kamiya N, Zhou X, Chen H, Hara T, Hoshi H, Yokoyama R, et al. Automated recognition of the psoas major muscles on X-ray $C T$ images. Conf Proc IEEE Eng Med Biol Soc. 2009;2009:3557-60.

49. Kamiya N, Zhou X, Chen H, Muramatsu C, Hara T, Yokoyama R, et al. Automated segmentation of psoas major muscle in X-ray CT images by use of a shape model: preliminary study. Radiol Phys Technol. 2012;5(1):5-14.

50. Yamashita M, Kamiya K, Matsunaga A, Kitamura T, Hamazaki N, Matsuzawa $\mathrm{R}$, et al. Prognostic value of psoas muscle area and density in patients who undergo cardiovascular surgery. Can J Cardiol. 2017;33(12):1652-9.

51. De Amorim BK, Bos SA, Veld J, Lozano-Calderon SA, Torriani M, Bredella MA. Body composition predictors of therapy response in patients with primary extremity soft tissue sarcomas. Acta Radiol. 2018;59(4):478-84.

52. Locke JE, Carr JJ, Nair S, Terry JG, Reed RD, Smith GD, et al. Abdominal lean muscle is associated with lower mortality among kidney waitlist candidates. Clin Transplant. 2017 Mar;31:3.

53. Veld J, Vossen JA, De Amorim BK, Halpern EF, Torriani M, Bredella MA. Adipose tissue and muscle attenuation as novel biomarkers predicting mortality in patients with extremity sarcomas. Eur Radiol. 2016;26(12):4649-55.

54. Zhang P, Peterson M, Su GL, Wang SC. Visceral adiposity is negatively associated with bone density and muscle attenuation. Am J Clin Nutr. 2015; 101(2):337-43.

55. Torriani M, Hadigan C, Jensen ME, Grinspoon S. Psoas muscle attenuation measurement with computed tomography indicates intramuscular fat accumulation in patients with the HIV-lipodystrophy syndrome. J Appl Physiol (1985). 2003 Sep;95(3):1005-10.

56. DiMartini A, Cruz RJ Jr, Dew MA, Myaskovsky L, Goodpaster B, Fox K, et al. Muscle mass predicts outcomes following liver transplantation. Liver Transpl. 2013;19(11):1172-80.

57. Lee CS, Cron DC, Terjimanian MN, Canvasser LD, Mazurek AA, Vonfoerster E, et al. Dorsal muscle group area and surgical outcomes in liver transplantation. Clin Transpl. 2014;28(10):1092-8.

58. Onuma T, Kamishima T, Shimamura T, Kawamura N, Yamashita K, Sutherland $K$, et al. Longitudinal CT study of sarcopenia due to hepatic failure after living donor liver transplantation. Quant Imaging Med Surg. 2018;8(1):25-31.

59. Canvasser LD, Mazurek AA, Cron DC, Terjimanian MN, Chang ET, Lee CS, et al. Paraspinous muscle as a predictor of surgical outcome. J Surg Res. 2014; 192(1):76-81.

60. Yong W, Xu B, Xiuping $T$, Junfeng Q. Paraspinal muscle segmentation in CT images using a single atlas. Progress in Informatics and Computing. 2015. https://doi.org/10.1109/PIC.2015.7489839.

61. Fortin $\mathrm{M}$, Omidyeganeh $\mathrm{M}$, Battié $\mathrm{MC}$, Ahmad $\mathrm{O}$, Rivaz H. Evaluation of an automated thresholding algorithm for the quantification of paraspinal muscle composition from MRI images. Biomed Eng Online. 2017;16(1):61.

62. Wei $Y$, Tao X, Xu B, Castelein A. Paraspinal muscle segmentation in CT images using GSM-based fuzzy C-means clustering. Journal of Computer and Communications. 2014. https://doi.org/10.4236/jcc.2014.29010.

63. Battaglia N V, Mahfouz M R, Johnson M. Semi-Automatic segmentation of the lumbar muscles and gender specific cross-sectional areas (Abstract). 58th Annual Meeting of the Orthopaedic. Research Society, San Francisco, CA, 2012

64. Otemuyiwa B, Derstine BA, Zhang P, Wong SL, Sabel MS, Redman BG, et al. Dorsal muscle attenuation may predict failure to respond to Interleukin-2 therapy in metastatic renal cell carcinoma. Acad Radiol. 2017 Sep;24(9):1094-100.

65. Weijs PJ, Looijaard WG, Dekker IM, Stapel SN, Girbes AR, Oudemans-van Straaten HM, et al. Low skeletal muscle area is a risk factor for mortality in mechanically ventilated critically ill patients. Crit Care. 2014;18(2):R12.

66. Englesbe MJ, Lee JS, He K, Fan L, Schaubel DE, Sheetz KH, et al. Analytic morphomics, core muscle size, and surgical outcomes. Ann Surg. 2012 Aug; 256(2):255-61.

67. Hyunkwang Lee, Fabian M T,Shahein T, Georg F,Julia M, Florian J F,Synho D. Artificial Intelligence Quantifies Muscle on Computed Tomography for Body Morphometric Analysis. J Digit Imaging. 2017; doi:https://doi.org/10.1007/ s10278-017-9988-z

68. Popuri K, Cobzas D, Esfandiari N, Baracos V, Jägersand M. Body composition assessment in axial CT images using FEM-based automatic segmentation of skeletal muscle. IEEE Trans Med Imaging. 2016;35(2):512-20. 
69. Silva de Paula N, de Aguiar BK, Azevedo Aredes M, Villaça Chaves G. Sarcopenia and skeletal muscle quality as predictors of postoperative complication and early mortality in gynecologic Cancer. Int J Gynecol Cancer. 2018:28(2):412-20.

70. Lee S, Paik HC, Haam SJ, Lee CY, Nam KS, Jung HS, et al. Sarcopenia of thoracic muscle mass is not a risk factor for survival in lung transplant recipients. J Thorac Dis. 2016 Aug;8(8):2011-7.

71. Derstine BA, Holcombe SA, Goulson RL, Ross BE, Wang NC, Sullivan JA, et al. Quantifying sarcopenia reference values using lumbar and thoracic muscle areas in a healthy population. J Nutr Health Aging. 2017;21(10):180-5.

72. Miller JA, Harris K, Roche C, Dhillon S, Battoo A, Demmy T, et al. Sarcopenia is a predictor of outcomes after lobectomy. J Thorac Dis. 2018 Jan;10(1):432-40

73. Fintelmann FJ, Troschel FM, Mario J, Chretien YR, Knoll SJ, Muniappan A, et al. Thoracic Skeletal Muscle Is Associated With Adverse Outcomes After Lobectomy for Lung Cancer. Ann Thorac Surg. 2018.

74. Karteek P, Dana C, Martin J, Nina E, Vickie B. Fem-based automatic segmentation of muscle and fat tissues from thoracic CT images. IEEE Trans Med Imaging. 2016;35(2):512-20.

75. Wajchenberg BL. Subcutaneous and visceral adipose tissue: their relation to the metabolic syndrome. Endocr Rev. 2000;21(6):697-738.

76. Guiu B, Petit JM, Bonnetain F, Ladoire S, Guiu S, Cercueil JP, et al. Visceral fat area is an independent predictive biomarker of outcome after first-line bevacizumabbased treatment in metastatic colorectal cancer. Gut. 2010;59(3):341-7.

77. Ladoire S, Bonnetain F, Gauthier M, Zanetta S, Petit JM, Guiu S, et al. Visceral fat area as a new independent predictive factor of survival in patients with metastatic renal cell carcinoma treated with antiangiogenic agents. Oncologist. 2011;16(1):71-81.

78. Ryo M, Kishida K, Nakamura T, Yoshizumi T, Funahashi T, Shimomura I. Clinical significance of visceral adiposity assessed by computed tomography: a Japanese perspective. World J Radiol. 2014;6(7):409-16.

79. Cakir H, Heus C, van der Ploeg TJ, Houdijk AP. Visceral obesity determined by $\mathrm{CT}$ scan and outcomes after colorectal surgery; a systematic review and meta-analysis. Int J Color Dis. 2015;30(7):875-82.

80. Demerath EW, Ritter KJ, Couch WA, Rogers NL, Moreno GM, Choh A, et al. Validity of a new automated software program for visceral adipose tissue estimation. Int J Obes. 2007 Feb;31(2):285-91.

81. Amol P, Alok NB, loannis AK. Application of computer vision. Appl Comp Vis. 2005. https://doi.org/10.1109/ACVMOT.2005.31.

82. Nemoto $M$, Yeernuer $T$, Masutani $Y$, Nomura $Y$, Hanaoka $S$, Miki $S$, et al. Development of automatic visceral fat volume calculation software for CT volume data. J Obes. 2014;2014:495084

83. Kim YJ, Lee SH, Kim TY, Park JY, Choi SH, Kim KG. Body fat assessment method using CT images with separation mask algorithm. J Digit Imaging. 2013;26(2):155-62.

84. Zhao B, Colville J, Kalaigian J, Curran S, Jiang L, Kijewski P, et al. Automated quantification of body fat distribution on volumetric computed tomography. J Comput Assist Tomogr. 2006;30(5):777-83.

85. Pan-Fu K, Kuo Y-L, Po-Tsun L, Wei-Chen C, Ya-Ling H, Chiun-li C. Fully automatic abdominal fat segmentation system from a low resolution $C T$ image. J Comput. 2015;36(2):65-77.

86. Lee SJ, Liu J, Yao J, Kanarek A, Summers RM, Pickhardt PJ. Fully automated segmentation and quantification of visceral and subcutaneous fat at abdominal CT: application to a longitudinal adult screening cohort. $\mathrm{Br}$ J Radiol. 2018:28:20170968.

87. Rosenquist KJ, Pedley A, Massaro JM, Therkelsen KE, Murabito JM, Hoffmann $U$, et al. Visceral and subcutaneous fat quality and cardiometabolic risk. JACC Cardiovasc Imaging. 2013;6(7):762-71.

88. Shah RV, Allison MA, Lima JA, Abbasi SA, Eisman A, Lai C, et al. Abdominal fat radiodensity, quantity and cardiometabolic risk: the multi-ethnic study of atherosclerosis. Nutr Metab Cardiovasc Dis. 2016;26(2):114-22.

89. Therkelsen KE, Pedley A, Rosenquist KJ, Hoffmann U, Massaro JM, Murabito $\mathrm{JM}$, et al. Adipose tissue attenuation as a marker of adipose tissue quality: associations with six-year changes in body weight. Obesity (Silver Spring). 2016;24(2):499-505.

90. Yeoh AJ, Pedley A, Rosenquist KJ, Hoffmann U, Fox CS. The association between subcutaneous fat density and the propensity to store fat viscerally. J Clin Endocrinol Metab. 2015;100(8):E1056-64.

91. Murabito JM, Pedley A, Massaro JM, Vasan RS, Esliger D, Blease SJ, et al. Moderate-to-vigorous physical activity with accelerometry is associated with visceral adipose tissue in adults. J Am Heart Assoc. 2015;4(3):e001379.
92. Rosenquist KJ, Massaro JM, Pedley A, Long MT, Kreger BE, Vasan RS, et al. Fat quality and incident cardiovascular disease, all-cause mortality, and cancer mortality. J Clin Endocrinol Metab. 2015;100(1):227-34.

93. Alvey NJ, Pedley A, Rosenquist KJ, Massaro JM, O'Donnell CJ, Hoffmann U, et al. Association of fat density with subclinical atherosclerosis. J Am Heart Assoc. 2014;3:4

94. Yong HS, Kim EJ, Seo HS, Kang EY, Kim YK, Woo OH, et al. Pericardial fat is more abundant in patients with coronary atherosclerosis and even in the non-obese patients: evaluation with cardiac CT angiography. Int J Cardiovasc Imaging. 2010;26(Suppl 1):53-62.

95. Jang HC, Lee HK, Lee H, Cha JG, Kim YS, Cho JH. Analyzing correlation between epicardial fat area and metabolic syndrome risk factor by using low-dose lung CT. Pak J Med Sci. 2015;31(5):1207-12.

96. Song DK, Hong YS, Lee H, Oh JY, Sung YA, Kim Y. Increased Epicardial adipose tissue thickness in type 2 diabetes mellitus and obesity. Diabetes Metab J. 2015;39(5):405-13.

97. Balci A, Celik M, Balci DD, Karazincir S, Yonden Z, Korkmaz I, et al. Patients with psoriasis have an increased amount of epicardial fat tissue. Clin Exp Dermatol. 2014;39(2):123-8.

98. Dagvasumberel M, Shimabukuro M, Nishiuchi T, Ueno J, Takao S, Fukuda D, et al. Gender disparities in the association between epicardial adipose tissue volume and coronary atherosclerosis: a 3-dimensional cardiac computed tomography imaging study in Japanese subjects. Cardiovasc Diabetol. 2012;11:106.

99. Oyama N, Goto D, Ito YM, Ishimori N, Mimura R, Furumoto T, et al. Singleslice epicardial fat area measurement: do we need to measure the total epicardial fat volume? Jpn J Radiol. 2011;29(2):104-9.

100. Maimaituxun G, Shimabukuro M, Fukuda D, Yagi S, Hirata Y, Iwase T, et al. Loca thickness of Epicardial adipose tissue surrounding the left anterior descending artery is a simple predictor of coronary artery disease - new prediction model in combination with Framingham risk score. Circ J. 2018;21.

101. Norlén A, Alvén J, Molnar D, Enqvist O, Norrlund RR, Brandberg J, et al. Automatic pericardium segmentation and quantification of epicardial fat from computed tomography angiography, 034003. J Med Imaging (Bellingham). 2016;3(3)

102. Rodrigues EO, Morais FFC, Morais NA, Coni LS, Neto LV, Conci A. A novel approach for the automated segmentation and volume quantification of cardiac fats on computed tomography. Comput Methods Prog Biomed. 2016;123:109-28.

103. Coppini G, Favilla R, Marraccini P, Moroni D, Pieri G. Quantification of Epicardial fat by cardiac CT imaging. Open Med Inform J. 2010;4:126-35.

104. Vladimir Z, Lidija K, Lazar V, Branislav P, Marko J, Ratko O, Nebojsa M. Semiautomatic Epicardial fat segmentation based on fuzzy c-means clustering and geometric ellipse fitting. Journal of Healthcare Engineering 2017. https://doi.org/10.1155/2017/5817970

105. Bandekar AN, Naghavi M, Kakadiaris IA. Automated pericardial fat quantification in CT data. Conf Proc IEEE Eng Med Biol Soc. 2006;1:932-5.

106. Abazida O. H Saqqaab. Epicardial fat quality effect on subclinical atherosclerosis. J Saudi Heart Assoc. 2016;28(3):129-220.

Ready to submit your research? Choose BMC and benefit from

- fast, convenient online submission

- thorough peer review by experienced researchers in your field

- rapid publication on acceptance

- support for research data, including large and complex data types

- gold Open Access which fosters wider collaboration and increased citations

- maximum visibility for your research: over $100 \mathrm{M}$ website views per year

At $\mathrm{BMC}$, research is always in progress.

Learn more biomedcentral.com/submission 